Expression of Concern

\title{
Expression of Concern on “Antigravity, an Answer to Nature's Phenomena including the Expansion of the Universe"
}

\author{
Advances in High Energy Physics \\ Received 21 September 2020; Accepted 21 September 2020; Published 17 April 2021 \\ Copyright (c) 2021 Advances in High Energy Physics. This is an open access article distributed under the Creative Commons \\ Attribution License, which permits unrestricted use, distribution, and reproduction in any medium, provided the original work \\ is properly cited.
}

Advances in High Energy Physics would like to express concern with the article titled "Antigravity, an Answer to Nature's Phenomena including the Expansion of the Universe" [1], which reviews the author's previous studies.

Following the publication of the review article, concerns have been identified that the discussion is qualitative and without any concrete model supporting the ideas presented. The concept that clouds experience anti-gravity proportional to the temperature of water droplets appears unsustainable, as well as the idea that thermal energy produced by the stars can explain the accelerating universe. Both ideas lack a concrete model, and it is unlikely that such a model can exist. If the observations presented in the article are assumed to be correct, a model would be required to test the hypothesis. The possibility of such testing is doubtful. We additionally note that the author's previous work, reviewed in the article, has not been cited by other researchers.

\section{References}

[1] C. K. Piyadasa Gamini, “Antigravity, an answer to Nature's phenomena including the expansion of the universe," Advances in High Energy Physics, vol. 2020, Article ID 9315491, 5 pages, 2020. 